Mikhail Z. Zgurovsky · Victor A. Sadovnichiy Editors

\title{
Continuous and Distributed Systems
}

Theory and Applications 


\section{Contents}

\section{Part I Abstract Algebra and Applications}

1 Algebra and Geometry Through Hamiltonian Systems. . . . . . . 3

Anatoly T. Fomenko and Andrei Konyaev

$1.1 \quad$ Introduction . . . . . . . . . . . . . . . . 3

1.2 Atoms and Their Symmetries................. 4

1.3 Integer Lattices of Action Variables for "Spherical

Pendulum" System . . . . . . . . . . . . . . . . . 7

1.4 Billiards in Confocal Quadrics . . . . . . . . . . . . . 10

1.5 Bertrand's Manifolds and Their Properties. . . . . . . . . 14

1.6 Lie Algebras with Generic Coadjoint Orbits

of Dimension Two . . . . . . . . . . . . . . . . . 17

References .......................... 19

2 On Hyperbolic Zeta Function of Lattices. . . . . . . . . . 23

L. P. Dobrovolskaya, M. N. Dobrovolsky, N. M. Dobrovol'skii and N. N. Dobrovolsky

$2.1 \quad$ Introduction . . . . . . . . . . . . . . . . 23

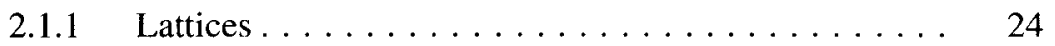

2.1.2 Exponential Sums of Lattices. . . . . . . . . 26

2.1.3 Multidimensional Quadrature Formulas

and Hyperbolic Zeta Function of a Grid . . . . . . . 29

2.1.4 Hyperbolic Zeta Function of Lattices . . . . . . . . 34

2.1.5 Generalised Hyperbolic Zeta Function of Lattices ... 40

2.2 Functional Equation for Hyperbolic Zeta Function

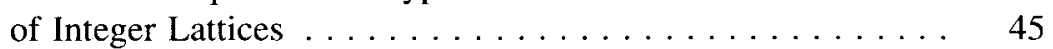

2.2.1 Periodized in the Parameter $b$ Hurwitz

Zeta Function. . . . . . . . . . . . . . . 46

2.2.2 Dirichlet Series with Periodical Coefficients ..... 47

2.2.3 Functional Equation for Hyperbolic Zeta Function of Integer Lattices . . . . . . . . . . . . . 50

2.3 Functional Equation for Hyperbolic Zeta Function

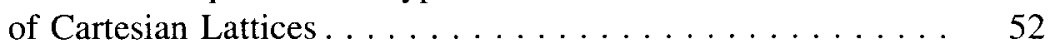


2.4 On Some Unsolved Problems of the Theory of Hyperbolic Zeta Function of Lattices . . . . . . . . . . . . 59

References ............................ 60

3 The Distribution of Values of Arithmetic Functions . . . . . . . . 63

G. V. Fedorov

References ........................... 66

4 On the One Method of Constructing Digital Control

System with Minimal Structure. . . . . . . . . . . . . . . 67

V. V. Palin

4.1 The Statement of Problem and Some Familiar Results . . . . . 67

4.2 Definitions and Some Preliminary Transformations . . . . . . . 68

4.3 The Method to Obtain the Characteristic

of Completely Controllable . . . . . . . . . . . . . . . . . . . 69

4.4 Auxiliary Statements. . . . . . . . . . . . . . . . . . . 69

4.5 The Absence of Associated Vectors Case . . . . . . . . . . . . . 70

4.6 The Case of General Position. . . . . . . . . . . . . . . . 71

Reference ........................... 71

5 On Norm Maps and "Universal Norms" of Formal Groups over Integer Rings of Local Fields. . . . . . . . . . . . . . . . . . . 73 Nikolaj M. Glazunov

$5.1 \quad$ Introduction $\ldots \ldots \ldots \ldots \ldots \ldots \ldots \ldots \ldots \ldots$

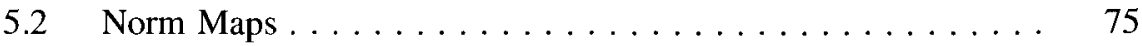

$5.3 \quad$ Results ....................... 78

References .......................... 80

6 Assignment of Factors Levels for Design of Experiments with Resource Constraints $\ldots \ldots \ldots \ldots \ldots \ldots$

S. A. Smirnov, A. A. Glushchenko, E. A. Ilchuk, I. L. Makeenko and N. A. Oriekhova

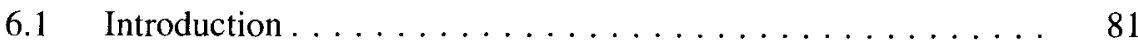

6.2 Hansel Method . . . . . . . . . . . . . . . . . . . 82

6.3 Modification ....................... 83

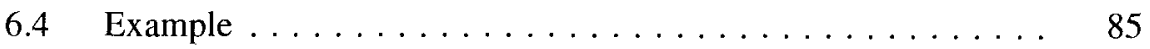

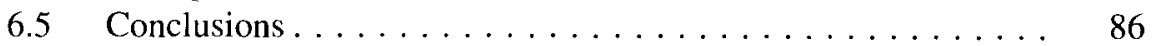

References ............................. 86 


\section{Part II Mechanics and Numerical Methods}

7 How to Formulate the Initial-Boundary-Value

Problem of Elastodynamics in Terms of Stresses? . . . . . . . . . . .

D. V. Georgievskii

7.1 The Classic Formulation of the Dynamic Problem and Its Peculiarities. . . . . . . . . . . . . . . . . . . . . . 89

7.2 Ignaczak-Nowacki' Formulation . . . . . . . . . . . . . . 91

7.3 Konovalov' Formulation . . . . . . . . . . . . . . . . . 92

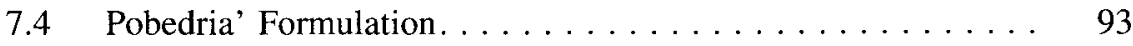

7.5 One More Possible Formulation . . . . . . . . . . . . . . . . . 93

References .............................. 95

8 Finite-Difference Method of Solution of the Shallow

Water Equations on an Unstructured Mesh. . . . . . . . . . . . . . . 97

G. M. Kobelkov and A. V. Drutsa

8.1 Introduction . . . . . . . . . . . . . . . . . . 97

8.2 Formulation of the Problem . . . . . . . . . . . . . . 97

8.3 Mesh and Mesh Operators . . . . . . . . . . . . . . . 98

8.4 Finite-Dimensional Problem. . . . . . . . . . . . . . . . . . . 100

8.5 Convergence . . . . . . . . . . . . . . . . . . . 101

8.6 Results of Numerical Experiments . . . . . . . . . . . . . . . . 104

8.6.1 Estimation of Convergence Order. . . . . . . . . . . 104

8.6.2 Computation of the Real Geographic Domain . . . . . 105

References ............................ 113

9 Dynamics of Vortices in Near-wall Flows

with Irregular Boundaries . . . . . . . . . . . . . . . . . 115

I. M. Gorban and O. V. Homenko

9.1 Introduction . . . . . . . . . . . . . . . . . . 115

9.2 Model of Standing Vortex . . . . . . . . . . . . . . . . . . 117

9.3 Standing Vortex in Cross Groove . . . . . . . . . . . . . . . 119

9.4 Standing Vortex in an Angular Region .............. 121

9.5 Resonant Properties of Standing Vortices

and Their Behavior in Perturbed Flow . . . . . . . . . . 123

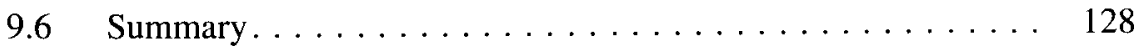

References ......................... 128 
10 Strongly Convergent Algorithms for Variational Inequality Problem Over the Set of Solutions the Equilibrium Problems

Vladimir V. Semenov

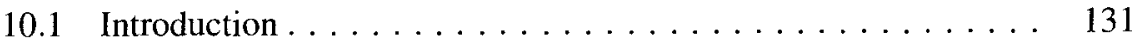

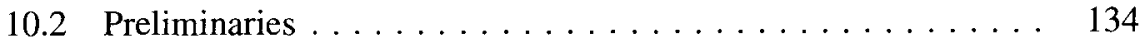

10.3 Convergence Analysis. . . . . . . . . . . . . . . 135

10.4 Concluding Remarks. . . . . . . . . . . . . . . . . . . . . 145

References . . . . . . . . . . . . . . . . . . . . . . . . . 145

Part III Long-time Forecasting in Multidisciplinary Investigations

11 Multivalued Dynamics of Solutions for Autonomous

Operator Differential Equations in Strongest Topologies . . . . . . 149

Mikhail Z. Zgurovsky and Pavlo O. Kasyanov

11.1 Introduction: Statement of the Problem . . . . . . . . . . . . 149

11.2 Additional Properties of Solutions . . . . . . . . . . . 151

11.3 Attractors in Strongest Topologies . . . . . . . . . . . . . . 158

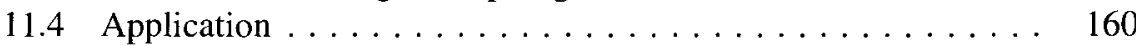

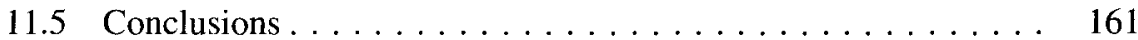

References ....................... 161

12 Structure of Uniform Global Attractor for General

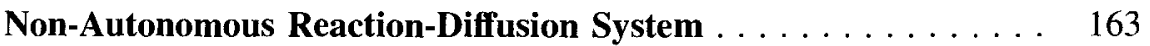

Oleksiy V. Kapustyan, Pavlo O. Kasyanov, José Valero and Mikhail Z. Zgurovsky

12.1 Introduction . . . . . . . . . . . . . . . . . . . . 163

12.2 Setting of the Problem ... . . . . . . . . . . . . . . 164

12.3 Multivalued Processes and Uniform Attractors. . . . . . . . . 165

12.4 Uniform Global Attractor for RD-System . . . . . . . . . . . 174

References ....................... 180

13 Topological Properties of Strong Solutions for the 3D

Navier-Stokes Equations

Pavlo O. Kasyanov, Luisa Toscano and Nina V. Zadoianchuk

13.1 Introduction . . . . . . . . . . . . . . . . . . . . . 181

13.2 Topological Properties of Strong Solutions . . . . . . . . . . 183

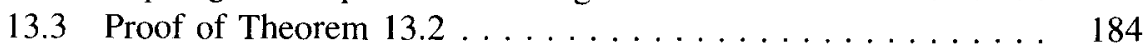

13.4 Proof of Theorem $13.1 \ldots \ldots \ldots \ldots \ldots \ldots \ldots$

References ......................... 187 
14 Inertial Manifolds and Spectral Gap Properties for Wave

Equations with Weak and Strong Dissipation . . . . . . . . . . . 189

Natalia Chalkina

14.1 Introduction . . . . . . . . . . . . . . . . . . . . . 189

14.2 Statement of the Problem and Spectrum of the Linear

Operator . . . . . . . . . . . . . . . . . . . 191

14.3 Sufficient Conditions for the Existence

of Inertial Manifolds . . . . . . . . . . . . . . . . . . 193

14.4 Proof of Theorem $14.3 \ldots \ldots \ldots \ldots \ldots \ldots$

14.4.1 New Norm in the Spaces $\mathscr{H}_{k}, k=1, \ldots, k_{1} \ldots \ldots 197$

14.4.2 New Norm in the Spaces $\mathscr{H}_{k}, k=k_{1}+1, \ldots, k_{2} \ldots 198$

14.4.3 New Norm in the Space $\mathscr{H}_{\infty} \ldots \ldots \ldots \ldots 200$

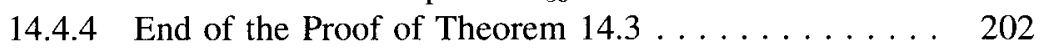

References ........................... 203

15 On Regularity of All Weak Solutions and Their Attractors

for Reaction-Diffusion Inclusion in Unbounded Domain . . . . . . . 205

Nataliia V. Gorban and Pavlo O. Kasyanov

15.1 Introduction . . . . . . . . . . . . . . . . . . . . . 205

15.2 On Compact Global Attractor for Reaction-Diffusion

Inclusion in Unbounded Domain . . . . . . . . . . . . . 208

15.3 Regularity of All Weak Solutions and Their Attractors . . . . . 217

References ... . . . . . . . . . . . . . . . . . . . . . 219

16 On Global Attractors for Autonomous Damped Wave

Equation with Discontinuous Nonlinearity . . . . . . . . . . . 221

Nataliia V. Gorban, Oleksiy V. Kapustyan, Pavlo O. Kasyanov

and Liliia S. Paliichuk

16.1 Introduction ...................... 221

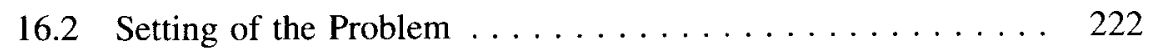

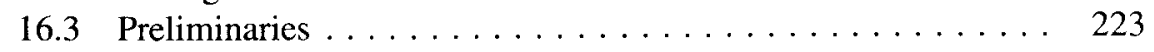

16.4 Properties of Solutions . . . . . . . . . . . . . . 225

16.5 The Existence of a Global Attractor . . . . . . . . . . 231

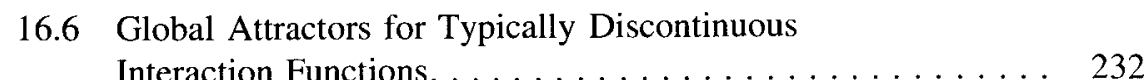

References ............................ 237

\section{Part IV Control Theory and Decision Making}

17 On the Regularities ofMass Random Phenomena. . . . . . . . . . 241

Victor I. Ivanenko and Valery A. Labkovsky

17.1 Introduction . . . . . . . . . . . . . . . . . . 241

17.2 Theorem of Existence of Statistical Regularities. . . . . . . . . 243

17.3 The Proof ...................... 246 
17.4 Applications in Decision Theory . . . . . . . . . . . . . . 247

17.5 Concluding Remarks. . . . . . . . . . . . . . . . . . . . 249

References ............................. 249

18 Optimality Conditions for Partially Observable Markov

Decision Processes . . . . . . . . . . . . . . . . . . . . 251

Eugene A. Feinberg, Pavlo O. Kasyanov and Mikhail Z. Zgurovsky

18.1 Introduction . . . . . . . . . . . . . . . . . 251

18.2 Model Description . . . . . . . . . . . . . . . . . . 252

18.3 Reduction of POMDPs to COMDPs and Optimality

Results ........................... 256

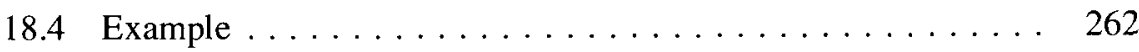

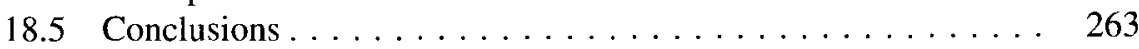

References ............................... 264

19 On Existence of Optimal Solutions to Boundary Control Problem for an Elastic Body with Quasistatic Evolution of Damage . . . . . 265

Peter I. Kogut and Günter Leugering

19.1 Introduction . . . . . . . . . . . . . . . . . . 265

19.2 Notation and Preliminaries. . . . . . . . . . . . . . . . . 266

19.3 Radon Measures and Convergence in Variable Spaces . . . . . 270

19.4 The Model of Quasistatic Evolution of Damage in an Elastic Material . . . . . . . . . . . . . . . . . . . 273

19.5 Setting of the Optimal Control Problems and Existence

Theorem for Optimal Traction . . . . . . . . . . . . . . 278

References ......................... 286

20 On Existence and Attainability of Solutions to Optimal Control Problems in Coefficients for Degenerate Variational Inequalities of Monotone Type . . . . . . . . . . . . . . . . . . . 287

Olga P. Kupenko

20.1 Introduction . . . . . . . . . . . . . . . . . . . . . . 287

20.2 Notation and Preliminaries. . . . . . . . . . . . . . . . . . 289

20.3 Setting of the Optimal Control Problem . . . . . . . . . . . . . 294

20.4 Compensated Compactness Lemma in Variable Lebesgue and Sobolev Spaces ... . . . . . . . . . . . . . . . . . 295

20.5 Existence of $H$-Optimal Solutions. . . . . . . . . . . . . . . 296

20.6 Attainability of $H$-Optimal Solutions . . . . . . . . . . . . 297

References . . . . . . . . . . . . . . . . . . . . . . . . . . 300 
21 Distributed Optimal Control in One Non-Self-Adjoint

Boundary Value Problem . . . . . . . . . . . . . . . . . 303

V. O. Kapustyan, O. A. Kapustian and O. K. Mazur

21.1 Introduction . . . . . . . . . . . . . . . . . . . 303

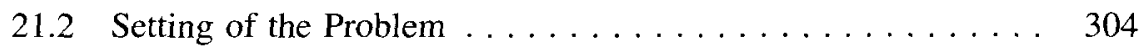

21.3 Main Results . . . . . . . . . . . . . . . . . . 305

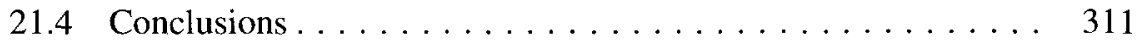

References ....................... 312

22 Guaranteed Safety Operation of Complex Engineering

Systems . . . . . . . . . . . . . . . . . . . . . 313

Nataliya D. Pankratova and Andrii M. Raduk

22.1 Introduction . . . . . . . . . . . . . . . . 314

22.2 Information Platform of Engineering Diagnostics

of the Complex Object Operation. . . . . . . . . . . 315

22.3 Diagnostic of Reanimobile's Functioning .......... 321

22.4 Conclusion. . . . . . . . . . . . . . . . . . . . . . . . . 325

References ............................ 326

Appendix A: To the Arithmetics of the Bose-Maslov

Condensate Statistics . . . . . . . . . . . . . . . . . . . . . . 327

Appendix B: Numerical Algorithms for Multiphase Flows

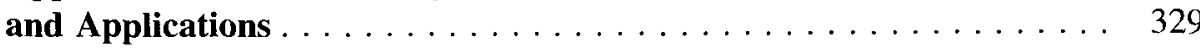

Appendix C: Singular Trajectories of the First Order in Problems with Multidimensional Control Lying

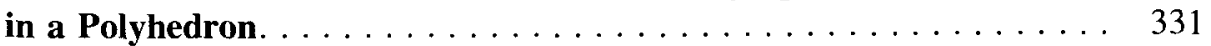

Appendix D: The Guaranteed Result Principle

in Decision Problems. 